\title{
Viszerale Thrombose
}

\section{Es droht der Darminfarkt!}

\author{
Eine viszerale Thrombose ist eine dramatische Erkrankung mit hoher Letalität. \\ Deshalb sollte bei jedem Patienten mit akuten starken Bauchschmerzen, für die sich \\ innerhalb von 24 Stunden keine andere Ursache finden lässt, diesem Verdacht \\ nachgegangen werden.
}

_ Mesenterialvenenthrombosen sind mit einer Inzidenz von 2,7/100.000 die häufigsten viszeralen Thrombosen. Dagegen treten Lebervenenthrombosen (Budd-Chiari-Syndrom) und Portalvenenthrombosen mit 1-4/1.000.000 deutlich seltener auf.

\section{Unspezifische Symptome, dramatische Verläufe}

Die Mesenterialvenenthrombose geht besonders häufig mit einem akuten $\mathrm{Ab}$ domen einher. Auch Übelkeit und Erbrechen können auftreten. Beim BuddChiari-Syndrom besteht bei etwa $70 \%$ der Patienten zusätzlich ein Aszites. Die Mesenterialvenenthrombose führt bei einem Viertel der Patienten $\mathrm{zu}$ einem Darminfarkt. In der gleichen Größenordnung bewegt sich die Frühletalität viszeraler Thrombosen. Das Rezidivrisiko beträgt $30 \%$ in fünf Jahren.

Eine akute Pfortaderthrombose verläuft in der Regel symptomatisch mit Bauchschmerz, Übelkeit und Diarrhö. Bei $20-40 \%$ der Patienten entwickeln sich untere gastrointestinale Blutungen. Treten eine Darmischämie und Multiorganversagen auf, versterben $20-50 \%$ der $\mathrm{Pa}$ tienten. Weniger ausgeprägt ist die Symptomatik, wenn sich die Portalvenenthrombose langsam entwickelt. Manchmal fällt sie dann erst auf, wenn eine portale Hypertension mit Aszites oder Ösophagusvarizen eingetreten ist.

\section{Risikofaktoren}

Für das Entstehen viszeraler Thrombosen gibt es lokale Risikofaktoren wie maligne Grunderkrankungen, Leberzirrhose, operative Eingriffe wie Cholezystektomie, entzündliche Prozesse oder ein stumpfes Abdominaltrauma. $\mathrm{Zu}$ den

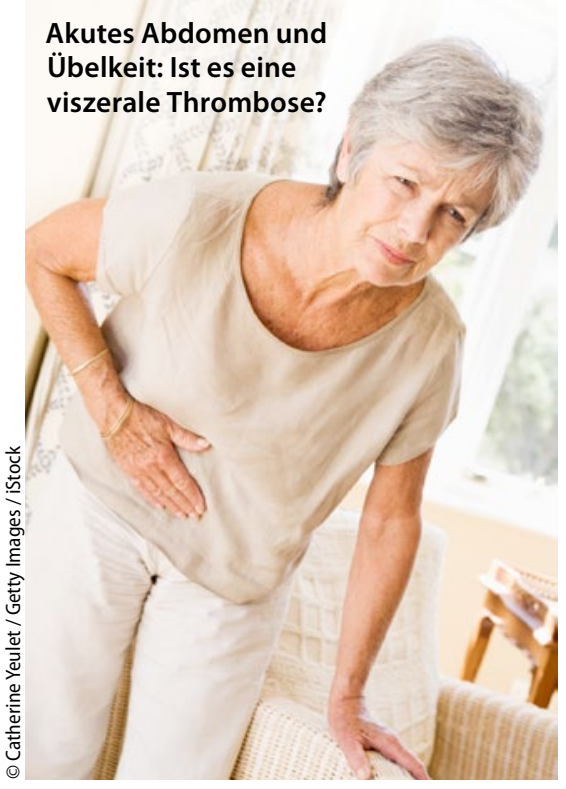

systemischen Risikofaktoren gehören Thrombophilie, Autoimmunerkrankungen, die Einnahme hormoneller Kontrazeptiva und Schwangerschaft.

\section{Antikoagulation: Ja oder Nein?}

Symptomatische Portalvenenthrombosen sollten ähnlich behandelt werden wie tiefe Beinvenenthrombosen, d.h. Antikoagulation für mindestens drei Monate, so Linnemann. Bei einer asymptomatischen Thrombose kann auf die Antikoagulation verzichtet werden, aber der Patient muss duplexsonografisch kontrolliert werden. Da jedoch auch 50-60\% der Patienten mit asymptomatischer Portalvenenthrombose persistierende Risikofaktoren aufweisen wie maligne Grunderkrankungen oder Leberzirrhose, wird dieses Vorgehen zunehmend angezweifelt.

Für die Antikoagulation spricht, dass diese oft zur Rekanalisierung des Gefä-

\section{Cave: myeloproliferative Erkrankung}

Relativ neu ist die Erkenntnis, dass man bei einer Viszeralvenenthrombose an eine myeloproliferative Erkrankung denken muss. Denn bei diesen Krankheitsbildern ist das Risiko für Viszeralvenenthrombosen um das 45-Fache erhöht. Nicht selten wird die myeloproliferative Erkrankung erst anlässlich einer Viszeralvenenthrombose diagnostiziert. Man sollte dann nach einer JAK2-Mutation suchen, die typisch für die essenzielle Thrombozytämie oder die Polyzytämia vera ist, betonte PD Dr. Birgit Linnemann, Universität Frankfurt. Patienten mit Viszeralvenenthrombose und JAK2-Mutation sollten frühzeitig einem Hämatoonkologen vorgestellt werden.

ßes führt und das Risiko für Komplikationen wie Darminfarkt sowie für portale Hypertension oder für Rezidive senkt. Dagegen spricht, dass es aus evtl. vorhandenen Ösophagusvarizen unter der Antikoagulation heftig bluten kann. Deshalb sollten Ösophagusvarizen vor einer Antikoagulation ligiert werden.

Die Leberzirrhose ist der wesentliche Risikofaktor für Thomboserezidive. Aber der Grat zwischen Thrombose und Blutungsrisiko wird mit fortschreitender Zirrhose zunehmend schmaler. Eine Sekundärprävention mit niedermolekularen Heparinen (NMH) bietet möglicherweise eine gute Alternative zur oralen Antikoagulation. Kleinere Studien haben gezeigt, dass NMH in Prophylaxedosierung das Risiko von Patienten mit fortgeschrittener Zirrhose und Portalvenenthrombose für Thromboserezidive, hepatische Dekompensation und Tod vermindern.

Dr. med. Angelika Bischoff

- Symposium "Atypische Thrombosen" DGIM-Kongress Mannheim, 11. April 2016 